\title{
STARSPOT MODELLING FROM BROADBAND \\ PHOTOMETRIC AND RADIO LIGHT CURVES
}

\author{
E. BUDDING \\ Carter Observatory, \\ P.O. Box 2909, Wellington, New Zealand.
}

\section{Introduction}

The concept of starspots or maculation is well established. There is indeed such a variety of corroborative evidence for those stars associated with 'activity' effects that we cannot imagine photospheric maculation not being a factor. The subject was reviewed by Eaton (1992), and a comprehensive background recently provided by Eker (1995), while relevant papers continue to be presented at more or less annual cool stars symposia. Even so, confidently testifying to more than the mere existence of starspots is non-trivial.

That the maculæ are predominantly cool regions can be asserted from numerous studies of the colour variation. Characteristic temperature differences between a starspot and the surrounding photosphere are of order 1000K (Vogt, 1981; Oláh, 1986; Strassmeier \& Oláh, 1992), i.e. comparable to those over sunspots with a substantial umbral component. Such results probe well the underlying effects (Eaton \& Hall, 1979; Byrne, 1992; Henry et al., 1995). Surface magnetic fields of order several thousand gauss are associated with such cooling (Saar, 1991).

Astronomy offers various instances of where the first and simplest type of model one can think of goes further in explanation than expected. Light curves which conform remarkably well to that produced by a simple black circular spot on a spherical stellar surface certainly exist, while two such spots seem to be able to reproduce most any typical maculation effect. But this must force on us the attention-demanding issue of uniqueness of the interpretation (Wild, 1991; Eker, 1995).

Eaton (1992) stressed progress in the areas of (i) the use of some standardized techniques for dealing with large bodies of data, particularly re- 
sulting from the use of APTs (e.g. Hall et al., 1986, Strassmeier et al., 1989), and (ii) the combination of spectroscopy and photometry to enhance the information from active stars. I would amplify these points in relation to (a) achieving higher data accuracy as a means to more reliable parametrization; (b) extending combinatorial techniques to beyond the optical domain, particularly with growing interest in stellar activity at radio, or high energy wavelengths; (c) applying reasonable inferences, so that some pictures become physically preferable to others, though all may be comparable in ability to produce satisfactory curve-fits.

\section{Active stars and starspots}

\section{1. $\mathrm{AB}$ DOR}

It is useful to have real examples to focus on. Innis et al. (1988) collected numerous data-sets on the well-known active star AB Dor. The distinct, well-defined periodicity to the maculation minima (Kubiak et al., 1995), together with repetitiveness to the general pattern of two minima per cycle, and certain other supportive data, suggested a 'weak pulsar' (or polar) picture of $\mathrm{AB}$ Dor, i.e. a rapidly rotating dwarf characterized by a predominating poloidal field, whose surface manifestation was as the two phase-locked spot minima (Vilhu et al., 1993). Such a field organization was considered in White et al.'s (1989) review. Doppler imaging techniques (e.g. Cameron, 1995) may well indicate much more complexity, but that is not pursued here. We want simply to ask, how far can photometric evidence go towards clarifying such a picture?

\subsubsection{The classical spot}

From time to time a light curve like that of Pakull (1981) (or Anders, 1990) appears. This simple type of light curve can be modelled with a single dark circular spot with a perhaps surprising degree of success (Banks \& Budding, 1990). Some definite parameter values can be inferred from this model, but much more could be expected if photometry could be significantly improved to beyond millimagnitude accuracy.

First, however, consider an example of rational inference as a discriminant. A light curve like the Pakull one can be modelled, with apparently fair precision, apart from a spot (zonal cap), by a dark sector bounded by two meridians of longitude, or a column of spots at various latitudes (Fig $1)$. It is hard to see, though, what physical cause would darken a sector. With the differential rotation expectable for a fluid star, nor could such an arrangement maintain itself for the time periods that the maculation waves persist for (cf. also Henry et al., 1995). 


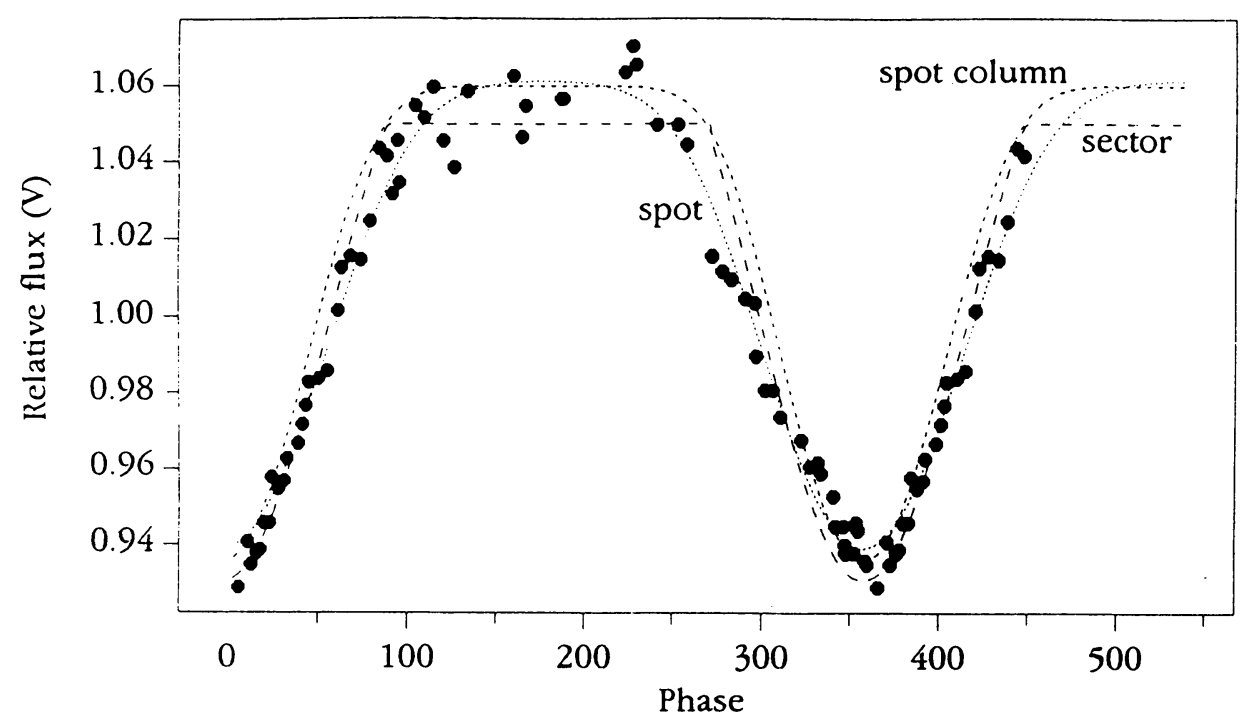

Figure 1. Alternative models are capable of feasibly fitting a given data-set.

In Figure 2 we show a series of curves showing the photometric effects of different parameters for the circular spot model (Budding, 1977). In each set of curves we keep the parameter set $\left\{\lambda=-5^{\circ}, \beta=45^{\circ}, i=70^{\circ}, \gamma=20^{\circ}\right.$, $\left.U=1, \kappa=0, L_{1}=1, u=0.8\right\}$, which is close to that for the Pakull (1981) one. The longitude $\lambda$, and unit of light $U$ were not changed, since $\lambda$ just moves the whole effect uniformly along in phase, while $U$ simply scales the ordinates to whatever out-of-minimum value is adopted. The effects show a separability to the parameters in the classical spot information-inversion problem. In a simple way, for inclination $i \neq 90^{\circ}$, and a light curve like Pakull's, the key parameters of $\lambda, \beta$ and $\gamma$ relate with the minimum's phase, width and depth, respectively. Colour variation can help find the right contrast factor $\kappa$, though a standard technique is to set $\kappa$ initially to zero to derive a minium spot area, and thence improve by successive approximations (cf. Zeilik et al., 1990). A single spot solution to the Pakull light curve with $i$ set to $70^{\circ}$ (Cameron et al., 1986; Banks \& Budding, 1990) results in: $\lambda=-2.4^{\circ}, \beta=50^{\circ}, \gamma=18.9^{\circ}$.

Here we find a 'high' latitude of $50^{\circ}$ preferred, as frequently reported from other comparable studies. Reasonable inferences from theory will support higher latitudes for spots on more rapidly rotating stars than the Sun 
(Schüssler \& Solanki, 1992). The surface eruption latitude in such rapidly rotating stars should not be less than arccos $[(R-D) / R]$, where $D$ is the depth of the convection zone, and $R$ is the stellar radius. With a value of $D \sim 0.35$ for AB Dor, eruption latitudes numerically less than $45^{\circ}$ should not be expected.

\subsubsection{Spot resolution}

Sometimes the spots of light curve modelling are interpreted as 'groups', like those associated with sunspots. Cameron \& Horne (1986), indicated that their technique might distinguish between a 'conglomerate' of small black dots and a single large 'monolith'. This could be understood if the subresolution spots had some clearly defined radiation law; otherwise, black dots could be replaced with grey areas expanding until they touched each other. Observed colour variations imply a relatively high 'umbral' fraction in the active areas, as mentioned before, but the question of resolution also arises here.

For a data-set having a photometric error measure $\delta l$, (as a proportion of full light) the smallest discernible spot has radius $\gamma$ of order $\sqrt{\delta l}$. For a maculation wave of amplitude $\Delta l$, a maximum number of spots to provide distribution information $N=\Delta l / \delta l$, typically $\sim 5$, might be suggested (but see below). The radius of a spot $\gamma$, whose area is effectively the same as that of a darkened region, can be specified to $\delta \gamma / \gamma \sim \delta l / 2 \Delta l$. Its longitude can be fixed to $\delta \lambda \sim \delta l / \sqrt{n} \Delta l$, where $n$ is the number of points associated with the minimum region of the light curve. $\delta \beta$ would be of order $\tan i \cos \beta \delta l$, from the width-latitude connection (Budding, 1977; Kjurkchieva, 1987; Eker, 1995). The effects of observational errors on parameter determination have recently been studied in detail by Kővári (1995); see also e.g. Aspin et al. (1981), and Rhodes et al. (1990).

\subsubsection{Fourier analysis}

The Pakull (1981) data was modelled by a Fourier series

$$
l=a_{0}+a_{1} \cos \left(\theta+\epsilon_{1}\right)+a_{2} \cos \left(2\left(\theta+\epsilon_{2}\right)\right)+a_{3} \cos \left(3\left(\theta+\epsilon_{3}\right)\right)+\ldots
$$

The coefficients of the higher order $\theta$ terms in this series dropped quite rapidly, and beyond the $3 \theta$ coefficient (itself 0.006 ) were appreciably below photometric accuracy. The series represented the data-set very well by retaining terms up to $4 \theta$, i.e. 9 independent parameters, but beyond that no significant improvement was found. Since 3 parameters are associated with a black circular spot, but another is needed to fix the reference light level, at the very most, 3 spots exhaust the information content of the data, and probably 2 spots come close. The Fourier transform of the theoretical curve due to a single spot is dominated by the lowest frequency components. In 

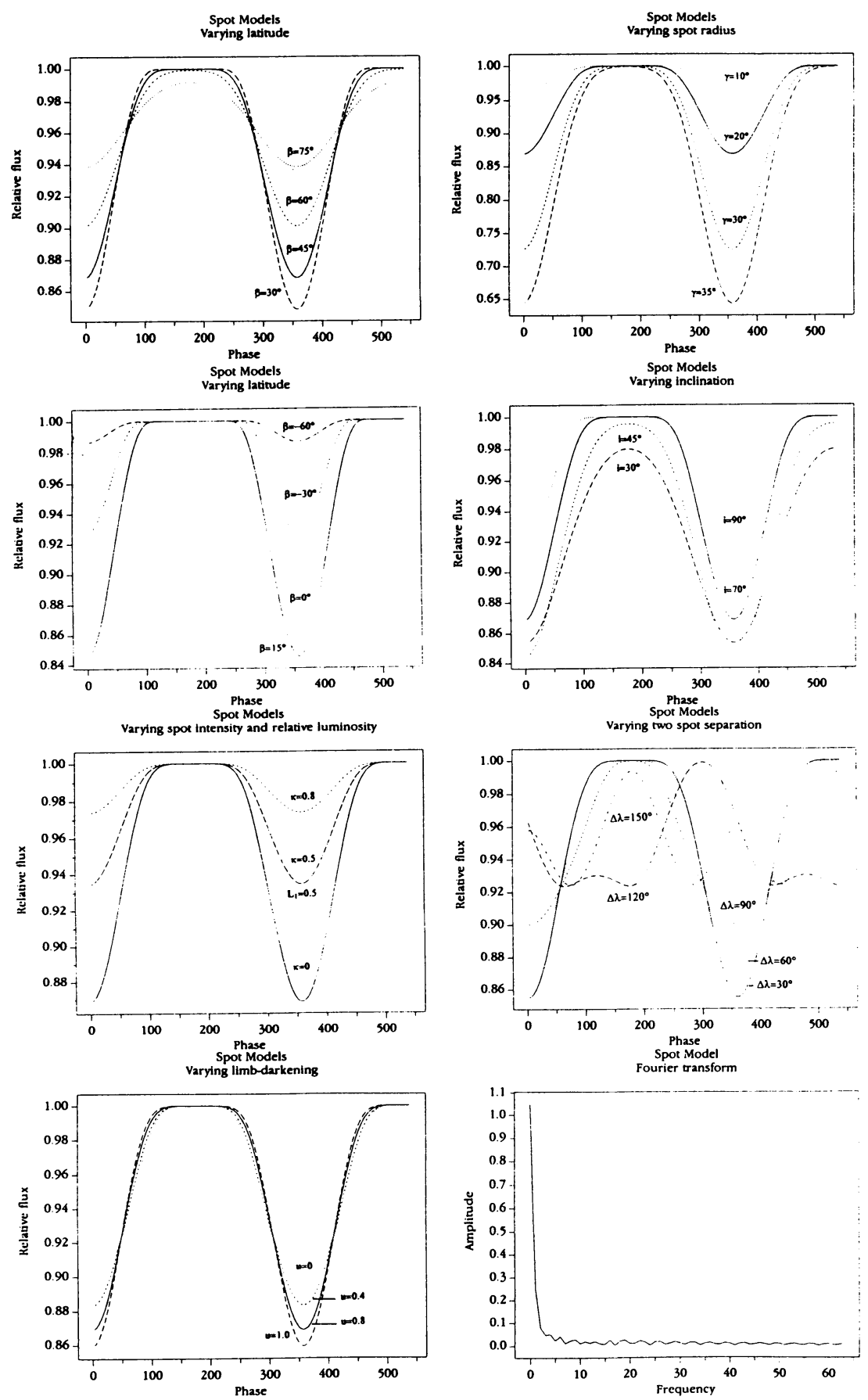

Figure 2. The effects of parameter variation for the circular starspot model (cf. also Strassmeier \& Bopp, 1992). 
fact, a single spot model for the Pakull data-set can be represented by a cosine series using only the two first terms in $\theta$ to an accuracy of $0.002 \mathrm{mag}$. That the Pakull data was to a small extent, but significant for the given observational error, improved by inclusion of the $3 \theta$ term, with a shifted phase, shows the single spot model to be a good, but not quite optimal, representation.

This relates to the approach of Hall et al. (1990), who used only terms in $\cos \theta$, thereby losing some parameter information. As an alternative, we replaced the single spot of Fig. 1 by a pair of the same area and latitude, allowing their separation freely to vary. Adding two equal amplitude cosine curves simply yields another, suitably scaled and displaced. Hall's formula is only the lower half of a complete sinusoid, even so the effect of two equal spots displaced by a longitude difference $\lesssim \pi / 3$ is qualitatively similar to varying the size, latitude or some other parameters for a single spot (cf. Fig. 2 ). Several tens of degrees is a typical spot pair resolution emerging from other analyses (Landstreet, 1992; Strassmeier \& Bopp, 1992; Strassmeier \& Oláh, 1992). In spot pair fittings to the Pakull (1981) data the latitude remained high $\left(>45^{\circ}\right)$, though it slowly declined as the maculated region was widened. The fitting deteriorated appreciably before $\beta$ fell below $45^{\circ}$, however.

The constant term also has physical interest, but contains no unambiguous positional information. In the following section on CF Tuc we will see how reasonable inferences can bear on this.

\subsubsection{Photometric picture of AB Dor}

A realistic error assessment then indicates that for the Pakull data-set the main, wave-causing macula has about the relative areal scale of Neptune's Dark Spot. It is likely to have a lot of concentrated umbra. This might be combined into one large group, or be a large pair separated by a longitude interval comparable to the diameter of the optimal single spot. But from most currently available photometric evidence alone we could not distinguish between a toroidal arrangement, in which spot pairs surface at particular preferred longitudes, and a poloidal picture. This dilemma will be resolved by increasing the time, spectral and accuracy coverage of this enigmatic star.

To illustrate the response of resolution to increasing data precision we present alternative fittings to the light curve of $A B$ Dor discussed by Cameron et al. (1986). The deeper minimum essentially aligns with the positive latitude spot at $\lambda \sim 100^{\circ}$. The secondary minimum associates with the negative latitude spot at longitude $290^{\circ}$. With a mean datum accuracy of 0.002 , that is all that can be fixed. The effect of Cameron et al.'s third spot, which bites into the ascending branch of the primary minimum $\left(\lambda \sim 130^{\circ}\right)$, 

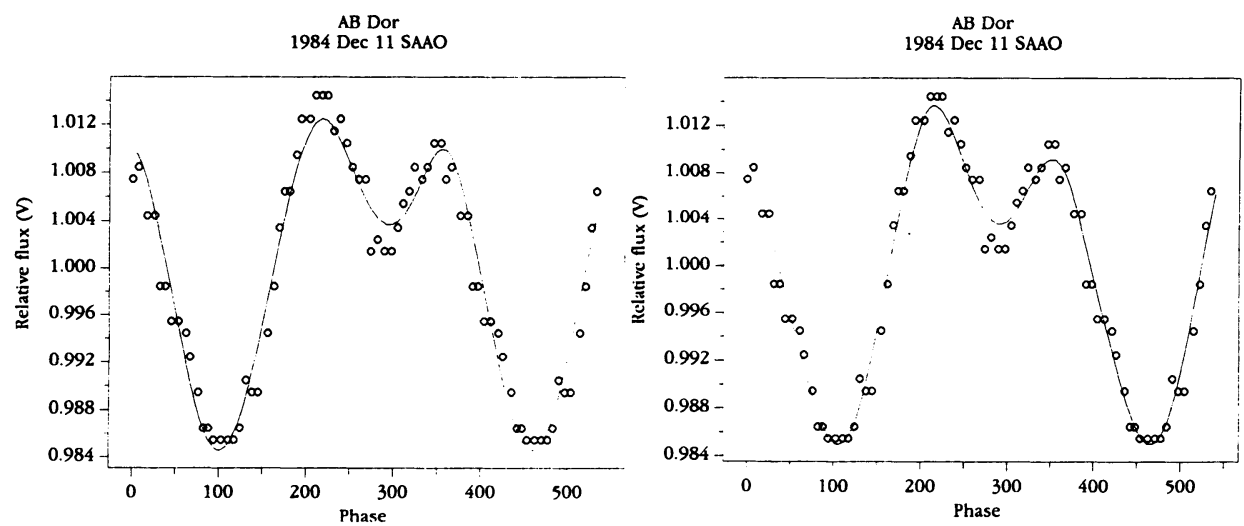

Figure 3. Two (l.h.) and three (r.h.) spot fittings to Cameron et al.'s (1986) data set. Three spots improve the fit, but require $\delta l \sim 0.0015$ in order to be significant.

can only be regarded as significant if photometric precision achieves 0.0015 . In fact, this third spot argues against the simple poloidal picture suggested by Vilhu et al. (1993). This points to the accuracy, and hence observing conditions, needed to improve the activity resolution of photometric data into a more productive research phase.

\subsection{CF TUC}

\subsubsection{RS CVn binaries}

These were reviewed by Ramsey (1990). Eclipsing RS CVn stars offer helpful factors for analysis over AB Dor, despite the proximity and other special points about the latter. This concerns the geometry and regular orbital revolution, though, in return, we have to distinguish the effects arising directly from binarity and maculation. Different procedures exist (cf. e.g. Kallrath, et al., 1992; Henry et al., 1995). In frequency terms, binarity has strong interdependent components at the fundamental orbital period and its low multiples. Eclipses introduce their own signatures, but these are all cosine terms, with zero phase shift for a classical close binary. There is also an underlying interdependence between proximity effects and eclipse parameters (cf. Kopal, 1979). In general, maculation centres at an arbitrary phase, and is much more dominated by low order cosine terms. 
Separation of the two effects can work as a sequential procedure. A preliminary close binary model is fitted to the raw light curve; the residue is fitted with a maculation curve, which is then added, with a negative sign, back to the original data; and a second 'cleaned' light curve fitted with an improved binary model (Budding \& Zeilik, 1987). The operation has normally been done satisfactorily, for available precisions, in one turn-round, but occasionally (e.g. the extreme case of XY UMa) a second correction stage was required. This technique has been applied to a number of datasets of RS CVn-like stars over the last several years.

Picking up now Eaton's (1992) extensive coverage point, some 25 datasets, mainly broadband $V$, of the RS CVn binary CF Tuc (Strassmeier et al., 1993; - CABS\#8), collected over a sixteen year period after 1978, were recently studied for maculation trends (Budding \& Zeilik, 1995). This relatively bright object has been monitored fairly regularly from small telescopes in Australasia (Anders et al., 1991; Rounthwaite, 1992). One good data-set (Budding \& McLaughlin, 1987) provided a 'template' eclipsing binary model. The idea concurs with the foregoing procedure: if the cleaned model really is that of an 'immaculate' system, then it should be generally valid. This approach is useful for data-sets which may be incomplete, or unsuitable for eclipse analysis, but still contain maculation information.

The activity of CF Tuc has been generally associated with the secondary, subgiant component of the binary (cf. Cameron et al., 1981, Coates et al., 1983a). To the available precision of many data-sets, photometric waves are often dominated by a single minimum, sometimes with a secondary feature. Effects can thus be modelled with one or two large starspots on the spectroscopically active secondary star. These again appear to be at higher latitudes, in comparison with the familiar solar case. One spot will often find itself at a longitude close to that of the central meridian at primary minimum (as with UX Ari, cf. Mohin \& Raveendran, 1989).

\subsubsection{Light curve trends}

Figure 4 shows maculation curve fittings for one subsection of the data, covering eight light curves, from the first one of Lloyd-Evans \& Koen (1987) for the end of 1978, and up to those of Coates et al. (1983b), from around mid-1982. The first four curves indicate a large spot persisting from the end of 1978 through 1979, but drifting back some 15-20 degrees in longitude.

The 1980 data of Cameron (1987) show a distinct new form to the wave. This cannot be from one large spot. The form is reproduced very faithfully by two large spots. A major darkening near opposition longitude is present, but the other spot finds itself close to second quadrature $\left(\sim 270^{\circ}\right)$. The nearopposition feature is at phase $\sim 380^{\circ}$, so, if this is a new spot, the old one continued to drift back in phase - a pattern repeated in subsequent data. 
That spot, hanging over from the first four light curves, must have drifted back at $\sim 40^{\circ}$ per year through 1980 .

The later curves from this period show a progressive diminution of the wave amplitude and a further drifting back of the minimum phase. The new spot predominates, drifting back some $50^{\circ}$ in longitude by mid- 1982 . The mean drift rate of spots turns out to be $\sim 30^{\circ}$ per year for these datasets. Differential features continued to decline through 1982, and into the generally featureless period of 83-4.

\subsubsection{Undistorted light curves 1983-84}

Solutions to the 'clean' eclipsing light curves of 1983 yielded $L_{2}$ to be about $37 \%$ of the whole, in the $V$ range. This is down by $25 \%$ on the clean solution to Cameron's light curve of 1980 . Moreover, the 1983 difference curves by Anders et al. (1991) show that the approximately constant out-of-eclipse level is down by $\sim 0.1 \mathrm{mag}$ from the brightest level of the star.

Four $20^{\circ}$ radius spots, at 90 deg intervals and 30 deg latitude, can reduce the overall brightness of the system by $0.1 \mathrm{mag}$, while the resulting light variation's amplitude is only 0.006 mag. The effective $L_{2}$ then drops by $20 \%$. The overall area of these spots is comparable to that of the fewer, highlatitude formations, which account for more distinct maculation waves. We can surmise then that the original large formations drift to lower latitudes and are gradually sheared or dispersed to a more even distribution in longitude (cf. Hall \& Busby, 1990). Asymmetries in this distribution account for any slight remaining variation.

\subsubsection{Photometric picture of CF Tuc}

There is an indication of a preferred longitude region for spot eruption close to the phase of primary minimum. This is different from the prevailing trends of the 'Short Period Group' (SPG) of RS CVn stars, where spotting most frequently occurs near the quadratures (Zeilik et al., 1990), but there may be some explanation involving binary geometry and magnetic buoyancy along the lines of Schüssler \& Solanki's (1992) discussion.

In the two or three years it takes for a new large spot to drift back to the phase of near second quadrature, another formation will typically have started to appear around the primary conjunction, as in 1980. Maculation effects then become quite complex, without a small number of dominant features or an 'active sector', as in 1983, and possibly 1988 and 1993. A near-central-meridian single spot later predominates again, thus starting off a new activity 'cycle'. The timescale for all this appears to be around $5-6$ years. Note this is a wave amplitude cycle - not necessarily the same as a mean luminosity cycle as considered by Anders et al. (1991) and others 

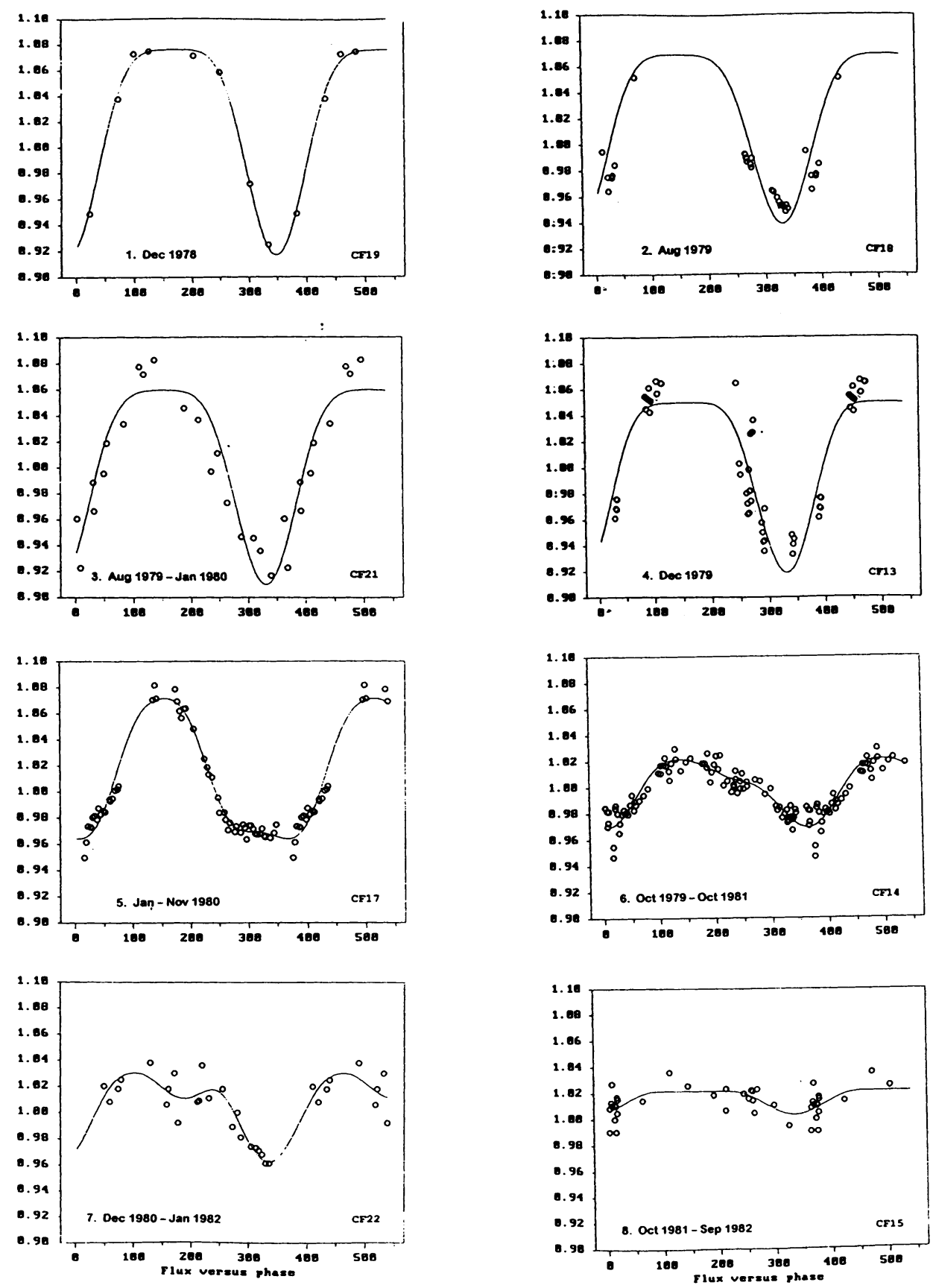

Figure 4. Maculation curves for CF Tuc for the period 1978-82. 
(e.g. Cutispoto \& Rodono, 1991), but it parallels the solar one, particularly with regard to the 'butterfly diagram' form (cf. e.g. Busso et al., 1986).

A new spot, at a latitude of $\sim \pm(40-50)^{\circ}$, drifts to lower phases at a rate of typically 30-50 deg/year, apparently drifting downward also in latitude during this process. This is in fair accord with expected migrations of spots arising from the differential rotation, using the data of Henry et al. (1995) for the coefficient appropriate to CF Tuc, though actual rates are not too constant in practice. Activity cycles in cool stars were reviewed by Baliunas \& Vaughan (1985), Linsky (1988) and Saar \& Baliunas (1992). Comparable photometric effects to the foregoing were reported for HK Lac (Oláh et al., 1991). The density of the outer atmosphere of CF Tuc's subgiant, up to an order of magnitude down on the corresponding region of the Sun, and corresponding magnetic flux values (cf. Saar, 1991) imply Alfvén velocities $v_{A}=2.2 \times 10^{11} / \sqrt{n_{e}} \mathrm{~cm} \mathrm{~s}^{-1}$ of perhaps $2-3$ times solar ones. The latitude range, from the trends of the longitude drift, is apparently restricted, so that an activity cycle timescale of about half that of the Sun is plausible.

\section{Radio light curves}

The importance of multiwavelength investigations of stellar activity has been increasingly recognized. By analogy with the Sun, the radio domain has the potential to probe an active region in its third or vertical dimension, thus complementing the two dimensional character of starspot analysis. The solar analogy has also led to the concept of the large emitting plasma loop, which is consistent with observations across wide wavelength ranges (Rosner et al., 1985; Stewart et al., 1988; Cameron \& Robinson, 1989).

Microwave emission from active stars is generally divided into a shortterm impulsive component, associated directly with flares, and a more longlasting (Lim et al., 1992) 'quiescent' component tending to undulate with a period comparable to that of the star's rotation. VLBI measurements have found bright 'core' regions within relatively large 'halo' structures having brightness temperatures (over stellar disk areas) in the range $10^{8}$ to several $\times 10^{10} \mathrm{~K}$, often with some circular polarization (Mutel et al., 1986; Lestrade et al., 1986, Slee et al., 1987). However, such large halos are not expected to relate to variations on the order of the orbital period or less, but form a background which slowly decays after an initial large flare. From the rotational modulation of earlier data, Slee et al., (1986) argued for quiescent source sizes of about $R_{*}$, a result consistent with the survey of Slee et al., (1988).

A broad correlation between X-rays and microwave data from active stars can be understood in terms of the loop model (White et al., 1990, Drake et al., 1989, 1992, Franciosini \& Chiuderi Drago, 1995), but the con- 
nection between the two emissions has given rise to much discussion. Such a correlation takes a thought-provoking form with the double-humped pattern seen for $\mathrm{AB}$ Dor in both X-ray and microwave regions contemporaneously (Vilhu et at., 1993). The analogy of a greatly scaled up solar active region (cf. Mullan, 1985) is also backed up by this pattern's anticorrelation with optical light variation from the maculæ (Slee et al., 1986). Such effects have been seen also for other stars, notably UX Ari (Elias et al., 1995), though period correlations were not always clear (cf. e.g. Chambliss et al., 1978). ${ }^{1}$ The role of smaller superposing flares also complicates interpretation (Ruciński, 1992), though more recent evidence can discriminate against some such contributions (Ruciński et al., 1993).

Gyrosynchrotron emission from a population of mildly relativistic electrons has often been seen as an underlying source for this quiescent emission (Dulk, 1987; Caillault et al., 1988; White et al., 1989, Morris et al., 1990), and the radio light curve of YY Gem, for example, was modelled in such terms (Gary, 1986). The exposition of Dulk (1985) is particularly helpful for such analyses. If we consider the temperatures required to explain the quiescent component in terms of active regions smaller than the stellar disk, however, $\left(T_{b} \gtrsim 10^{11}\right)$ the corresponding Lorentz factor $(\gamma \gtrsim 10)$ takes us into a fully relativistic regime, in which the emission concentrates into a small cone about the direction of instantaneous motion of an electron. This possibility has been examined in connection with the quiescent microwave emission from AB Dor by Lim et al., (1994). The optical depth $\tau$ of a path length $l$ is expressed, in the synchrotron case, using Dulk's (1985) formula (41) (symbols have conventional meanings), by

$$
-19.7+\log l+\log N_{e}+2.5 \log B-3.5 \log \nu_{G}=\log \tau,
$$

A representative power-law distribution of source electrons has been adopted here, with index $\delta=3$ and $\sin \theta=\pi / 4$. With $l=A r_{\text {spot }}$, where $r_{\text {spot }}$ represents a minimum effective cross sectional length for a flux loop above the active region and $A$ is an aspect factor $\sim 1$, we can plot lines of constant $\tau$ in the $N_{e}: B$ plane for various microwave frequencies. Keeping in mind AB Dor, we set $l=10^{10} \mathrm{~cm}, \tau=1$ for such lines in Figure 5. The argument here is that we 'see down' to a region which quickly becomes fully opaque (cf. Stewart et al., 1988). We plot in this same diagram the lines $\nu=\nu_{s}$, corresponding to the Razin-Tsytovich suppression condition, and also some coronal model lines. These show an $N_{e}(R)$ function of Baumbach type, with different base electron densities $\left(N_{e 0}\right)$. At a given value of

\footnotetext{
${ }^{1}$ The hump around phase 0.5 in Gunn et al.'s (1995) radio light curves of CF Tuc anticorrelates with a near-contemporaneous maculation minimum (Budding \& Zeilik, 1995).
} 
$R \equiv R^{-1}\left(N_{e}\right)$ the field is $B_{0}\left(R / R_{d}\right)^{-3}$, or a simple dipole decline, thereby mapping $N_{e}$ with $B$ along a given model. Photospheric field strengths of order several $\times 10^{3}$ gauss are observed for active stars (Saar, 1991). We have assumed a 'buried dipole', set the field at the base of the corona to be 3000 gauss, and put $R_{d}=r_{\text {spot }}$ for Figure 5 .

For an optically thick synchrotron source of characteristic size $L$ we have

$$
\log L=8.5-\log \nu_{G}-\log \sin \psi+0.5\left(\log \mathcal{L}-\log T_{\text {eff }}\right),
$$

where $\mathcal{L}$ is the luminosity in $\operatorname{erg~sec}^{-1} \mathrm{~Hz}^{-1}$ and the $\sin \psi$ factor $(\sim 1 / \gamma)$ allows for the emission's directivity. Dulk's (1985) formula (42) enables us to substitute for $T_{\text {eff }}$ in terms of $\nu_{G}$ and $B$, and thence the condition of source optical thickness, i.e. $L$ at least as great as the absorption length $l$, can also be plotted in the $N_{e}: B$ plane by combining (2) and (3) for a given flux level and distance. The $l=10^{10} \mathrm{~cm}$ lines, with $\log \mathcal{L}=16$ (Mutel et al., 1987), lie close to this condition in Figure 5, thus supporting the picture of an $\sim r_{\text {spot}}$-sized source just becoming optically thick at typical observed luminosity levels. The Razin-Tsytovich effect implies that appropriate electron densities are lower than for the solar corona.

An interpretation of the undulating, quasi-periodic term in microwave emission now becomes possible in terms of the varying aspect with phase of a large loop containing high energy electrons confined by large pitch angles. Different frequencies scan different positions across this structure, so that the undulation amplitude would vary, but the combination of effective area and different inclinations of the line of sight to the main emission direction causes a sensitive dependence of amplitude on frequency in the case of synchrotron emission. At intermediate microwave frequencies, if similar areas of the prominence were compared, the effective temperature variation in Dulk (1985) suggests a flat, or slightly negative gradient to the spectrum (Figure 5 combined with Dulk (42) for a constant size source and the same emission geometry yields $\alpha=-0.09$ ). The low circular polarization of such radio light curves (e.g. Ruciński, 1992, Lim et al., 1994) are consistent with this optically thick scenario.

A general formula for the radio variation can be simply cast in the form

$$
f=a_{b}(\nu)+a_{u}(\nu) \sigma\left(u, \gamma, z_{0}^{\prime}\right),
$$

where $a_{b}(\nu), a_{u}(\nu)$ are background and undulating components to the emission, $\sigma$ is the normal 'spot' function (Budding, 1977), $u$ is a limb-variation coefficient, but we have written $z_{0}^{\prime}$ to represent a more generalized aspect co-ordinate. Thus, 'top' (r), 'side' ( ) and 'end' ( ) projections could be involved for an isotropic source. For a source with relativistic directivity we could write $z_{0}^{\prime}=\left(z_{0}-z_{1}\right) /\left(1-z_{1}\right)$, where $z_{1}$ refers to the beam concentration toward the line of sight, i.e. $z_{1}=\cos \psi$. 


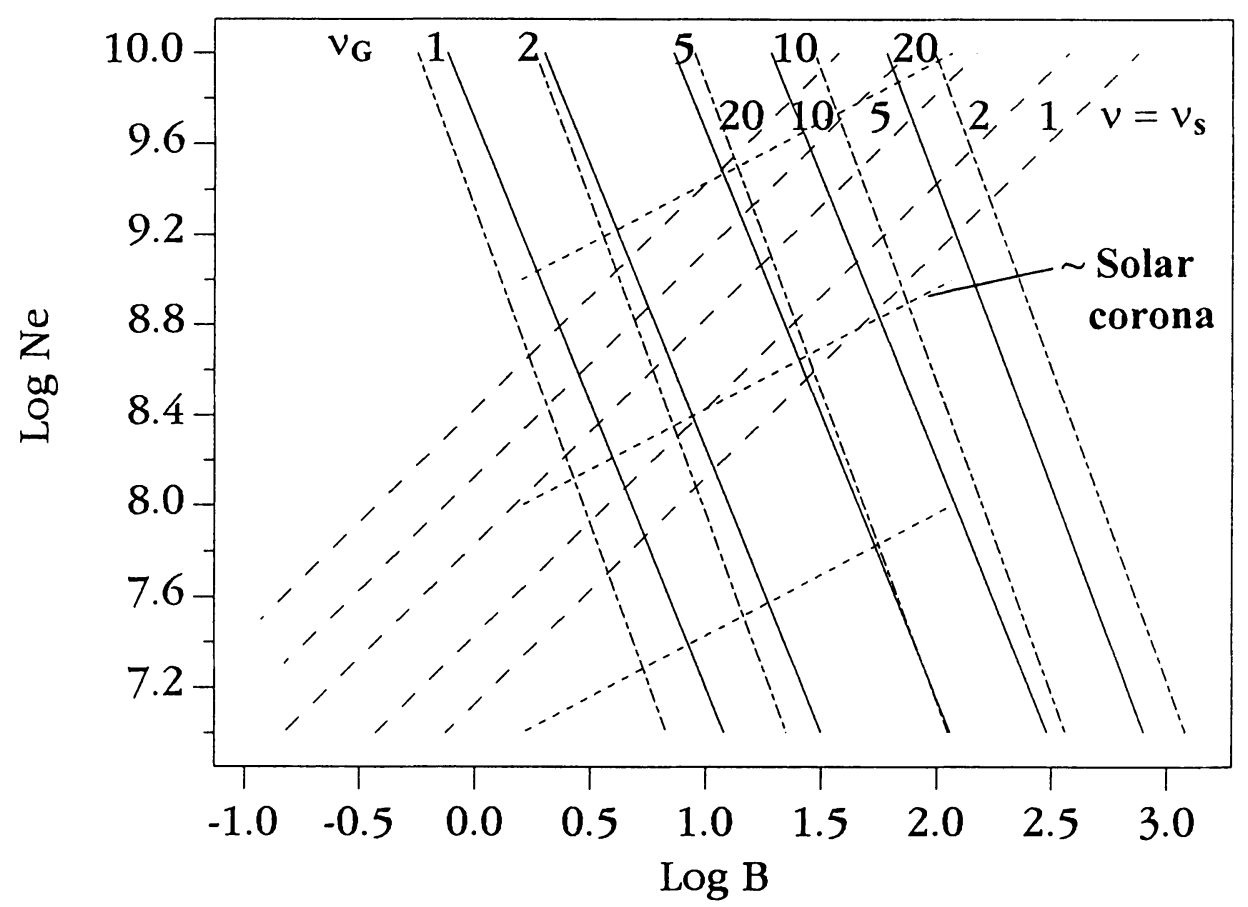

Figure 5. Lines of optical depth $\tau=1$ (full), Razin-Tsytovich suppression (long-dash), condition of optical thickness for apparent luminosity $\log \mathcal{L}=16$ (dot-dash) and coronal models with base electron density $N_{e 0}=10^{8}, 10^{9}$ and $10^{10}$ (short-dash).

In Figure 6, formula (4) has been applied to the $6 \mathrm{~cm}$ light curve of $\mathrm{AB}$ Dor, observed by Lim et al. (1994) on Oct 1, 1990. This diagram is mainly intended to demonstrate feasibility of the approach, but the curve-fitting exercise allowed insights into a possible information yield. For example, if the beaming factor $(\psi)$ and peak intensity of the emission can be alternatively constrained from reasonable theory, the fitting could provide a sensitive test for the latitude of the active region.

\section{Conclusions}

This paper has aimed at the resolution of stellar active regions by optical photometry and microwave radiometry. The analysis of AB Dor revealed the information limits of photometric analysis, but also showed that if precision could significantly advance beyond the millimag level key model discriminants, e.g. maculation latitudes or effective number of active regions, could be accessed. If complete and accurate light curves can be continuously gathered within 10-20 orbital periods, an effective way of probing activity 
AB Dor

$6 \mathrm{~cm}$ light curve feature

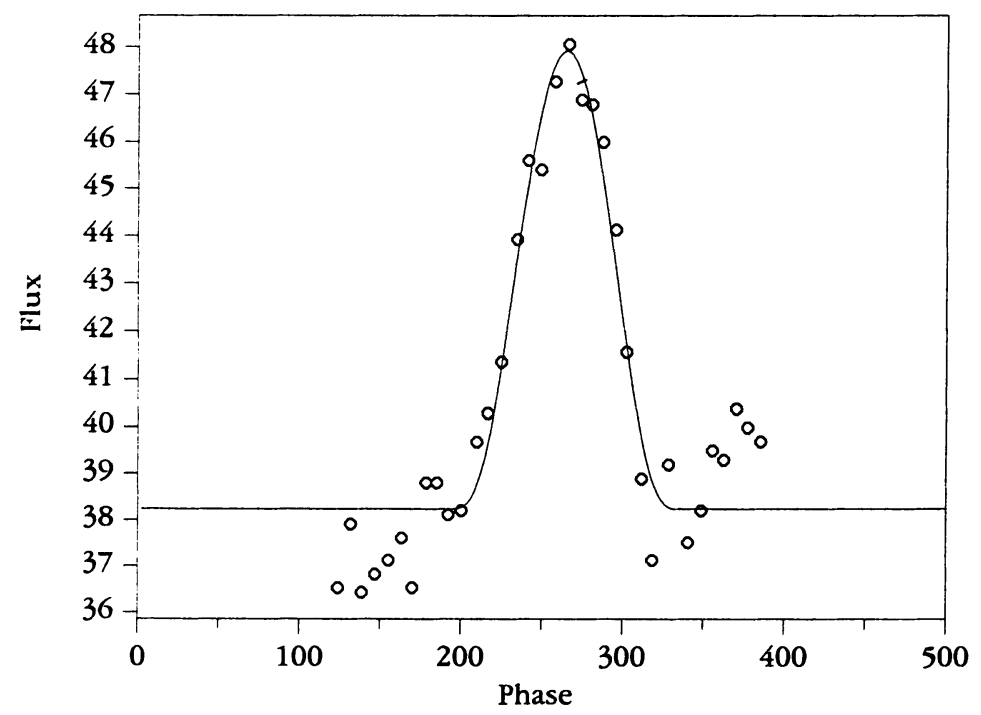

Figure 6. $\sigma$-function fitting to the 'wave' feature in the $6 \mathrm{~cm}$ light curve of AB Dor.

characteristics of stars like CF Tuc was also indicated. There seem to be simplifying gross factors, such as preferred longitudes, which grant relevance to simple models (e.g. large circular spots) for parametrizing photometric data, though this interpretation, on purely photometric grounds, is not unique (cf. also e.g. Hilditch \& Bell, 1994; Eaton et al., 1995). Progress can be expected, however, from applying combinatorial techniques, both observationally (e.g. using Doppler or Zeeman imaging at optical frequencies, as well as UV and X-ray information) and theoretically. An important issue in data analysis is to assess the level of parameter determinacy in relation to the inherent precision of a data-set. Against such a framework we can speak of the adequacy, merit, or even optimality, of a given characterization.

Microwave radiometry plays a complementary role in portraying the vertical structure of active regions. Key quantities, such as loop dimensions, interrelate to photometric analysis. Alternatively, the radio light curves may themselves prove to offer sensitive determinants of geometrical parameters. Such detailed complementarity of study is only recently organized. The required sensitivities of radiometry also imply that data may only be productive towards detailed modelling from a small number of relatively nearby stars. Increasing development of VLBI techniques may allow further clarifications, particularly, one might hope, for AB Dor, and also CF Tuc, where 'eclipse-imaging' may also become applicable (Gunn et al., 1995). 


\section{References}

Anders, G., 1990, Inf. Bull. Var. Stars \#3437.

Anders, G. J., Coates, D. W. \& Thompson, K., 1991, Vistas Astron., 34, 291.

Aspin, C., Simmons, J. F. L. \& Brown, J. C., 1981, MNRAS, 194, 283.

Baliunas, S. L. \& Vaughan, A. H., 1985, ARA $6 A$, 23, 379.

Banks, T. \& Budding, E., 1990, Ap\&SS, 167, 221.

Budding, E., 1977, Ap\&SS, 48, 207.

Budding, E., \& McLaughlin, E., 1987, Ap\&SS, 133, 45.

Budding, E. \& Zeilik, M., 1987, ApJ, 319, 827.

Budding, E. \& Zeilik, M., 1995, Ap\&SS., (in press).

Busso, M., Scaltriti, F. \& Cellino, A., 1986, $A \mathscr{E} A$, 156, 106.

Byrne, P. B., 1992, in Surface Inhomogeneities on Late-Type Stars, eds. P. B. Byrne \& D. J. Mullan, Springer, Lect. Notes Phys., 397, 3.

Caillault, J. P., Drake, S. \& Florkowski, D., 1988, $A J, 95,887$.

Cameron, A. C., Hearnshaw, J. B. \& Austin, R. R. D., 1981, MNRAS, 197, 769.

Cameron, A. C., Bedford, D. K., Evans, T. L., Ruciński, S. M., Vilhu, N. E. \& White, N. E., 1986, in Cool Stars, Stellar Systems, and the Sun, ed M. Zeilik \& D. M. Gibson, Springer, Lect. Notes Phys., 254, p. 313.

Cameron, A. C., \& Horne, K. D., 1986, in Cool Stars (etc. ibid.), 205.

Cameron, A. C., 1987, S. Afr. Astron. Obs. Circ., 11, 57.

Cameron, A. C. \& Robinson, R. D., 1989, MNRAS, 238, 657.

Cameron, A. C., 1995, MNRAS, (preprint).

Chambliss, C. R., Hall, D. S., Landis, H. J., Louth, H., Olson, E. C., Renner, T. R., Skillman, D. R., 1978, AJ., 83, 1514.

Coates, D. W., Halprin, L., Sartori, P. A. \& Thompson, K., 1983a, MNRAS, 202, 427.

Coates, D. W., Innis, J. L. \& Thompson, K., 1983b, Inf. Bull. Var. Stars, \#2779.

Cutispoto, G. \& Rodono, M., 1992 in Surface Inhomgeneities on Late Type Stars, eds. P. B. Byrne \& D. J. Mullan, Springer, Lect. Notes Phys., 397, 267.

Drake, S. A., Simon, T., \& Linsky, J. L., 1989, ApJS, 71, 905.

Drake, S. A., Simon, T., \& Linsky, J. L., 1992, ApJS, 82, 311.

Dulk, G. A., 1985, ARA \&A., 23, 169.

Dulk, G. A., 1987, Adv. Space Res., 6, 95.

Eaton, J. A., 1992, in Surface Inhomgeneities on Late Type Stars, eds. P. B. Byrne \& D. J. Mullan, Springer, Lect. Notes Phys., 397, 15.

Eaton, J. A. \& Hall, D. S., 1979, ApJ, 227, 907.

Eaton, J. A., Henry, G. W. \& Fekel, F. C., 1995, ApJ Lett., (preprint).

Eker, Z., 1995, ApJ, 445, 526.

Elias, N. M., Quirrenbach, A., Witzel, A., Naundorf, C. E., Wegner, R., Guinan, E. F. \& McCook, G. P., 1995, ApJ, 439, 983.

Franciosini, E. \& Chiuderi Drago, F., 1995, $A \& A, 297,535$.

Gary, D. E., 1986, in Cool Stars (etc.), see Cameron et al. 1986, above, p. 235.

Gunn, A. G., Migenes, V., Doyle, J. G. \& Spencer, R. E., 1995, MNRAS., (preprint).

Hall, D. S. \& Busby, M. R., 1990, in Active Close Binaries, ed. C. İbanoğlu, Kluwer, p377.

Hall, D. S., Fekel, F. C., Boyd, L. J. \& Genet, R. M., 1986, in Cool Stars (etc.), see Cameron et al. 1986, above), p. 64.

Henry, G. W., Eaton, J. A., Hamer, J. \& Hall, D. S., 1995, ApJ, (preprint).

Hilditch, R. W. \& Bell, S. A., 1994, MNRAS, 267, 1081.

Innis, J. L., Coates, D. W. \& Thompson, K., 1988, MNRAS, 233, 887.

Kallrath, J., Milone, E. F. \& Stagg, C. R., ApJ, 389, 590.

Kopal, Z., 1979, Language of the Stars, Reidel.

Kövári, Zs., 1995, poster at this conference.

Kjurkchieva, D. P., 1987, ApESSS, 138, 141.

Kubiak, M., Stepień, K., Kiraga, M. \& Jahn, K., 1995, Acta Astron., 45, 279. 
Landstreet, J. D., 1992, A\&A Rev., 4, 35.

Lestrade, J-F., Mutel, R. L., Preston, R. A., Phillips, R. B., 1986, in Cool Stars (etc.), see Cameron et al. 1986, above, p. 135.

Lim, J., Nelson, G. J., Castro, C., Kilkenny, D. \& van Wyk, F., 1992, ApJ, 388, L27.

Lim, J., White, S. M., Nelson, G. J. \& Benz, A. O., 1994, ApJ, 430, 332.

Linsky, J. L., 1988, in Multiwavelength Astrophysics, ed. F. Cordova, Cambr. Univ. Press, p. 49.

Lloyd-Evans, T. \& Koen, M. C. J., 1987, S. Afr. Astron. Obs. Circ., 11, 21.

Mohin, S. \& Raveendran, A. V., 1989, J. Astrophys. Astron., 10, 35.

Mutel, R. L., Lestrade, J. F., Preston, R. A. \& Phillips, R. B., 1986, ApJ, 289, 262.

Mutel, R. L., Morris, D. H., Doiron, D. J. \& Lestrade, J.-F., 1987, AJ, 93, 1220.

Morris, D. H., Mutel, R. L. \& Su, B., 1990, ApJ, 362, 299.

Mullan, D. J., 1985, in Radio Stars, eds. R. M. Hjellming \& D. M. Gibson, Reidel, 173.

Oláh, K, 1986, Commun. Konkoly Obs., 86, 393.

Oláh, K., Hall, D. S., \& Henry, G. W., 1991, $A \mho A, 251,531$.

Pakull, M. W., 1981, $A \& A, 104,33$.

Ramsey, L. W.., 1990 in Cool Stars, Stellar Systems and the Sun, ed. G. Wallerstein, $P A S P C, \mathbf{9}, 195$

Rhodes, M., Budding, E. \& Zeilik, M., 1990, in Cool Stars, Stellar Systems and the Sun, ed. G. Wallerstein, $P A S P C, \mathbf{9}, 252$.

Rosner, R., Golub, L., \& Vaiana, G. S., 1985, Ann. Rev. A\&A, 23, 413.

Rounthwaite, T., 1992, South. Stars, 34, 392.

Ruciński, S. M., 1992, PASP, 104, 1177.

Ruciński, S. M., Krogulec, M., \& Seaquist, E. R., 1993, AJ, 105, 2308.

Saar, S. H., 1991, in The Sun and Cool Stars:, eds. I. Tuominen, D. Moss \& G. Rüdiger, Springer, Lect. Notes Phys., 380, 389.

Saar, S. H. \& Baliunas, S. L., 1992, Proc. 4th Solar Cycle Workshop, ed. K. Harvey, $P A S P C, \mathbf{2 7}, 150$.

Schüssler, M. \& Solanki, S. K., 1992, $A \& A$, 264, L13.

Slee, O. B., Nelson, G. J., Innis, J. L., Stewart, R. T., Vaughan, A. E. \& Wright, A. E, 1986, Proc. Astron. Soc. Australia, 6, 312.

Slee, O. B., Nelson, G. J., Stewart, R. T., Wright, A. E., Innis, J. L., Ryan, S. G. \& Vaughan, A. E., 1987, MNRAS, 229, 659.

Slee, O. B., Stewart, R. T., Nelson, G. J., Wright, A. E., Dulk, G. A., Bastian, T. S. \& McKean, M., 1988, Astrophys. Lett. Commun., 27, 247.

Stewart, R. T., Innis, J. L., Slee, O. B., Nelson, G. J., Wright, A. E., 1988, AJ, 96, 371.

Strassmeier, K. G., Hall, D. S., Boyd, L. J., Genet, R. M., 1989, ApJS, 69, 141.

Strassmeier, K. G. \& Bopp, B. W., 1992, $A \& A, \mathbf{2 5 9}, 183$.

Strassmeier, K. G. \& Oláh, K., 1992, $A \& A, 259,595$.

Strassmeier, K. G., Hall, D. S., Fekel, F. C., \& Scheck, M., 1993, A\&AS, 100, 173.

Vilhu, O., Tsuru, T., Cameron, A. C., Budding, E., Banks, T., Slee, B., Ehrenfreund, P. \& Foing, B. H., 1993, $A \& A, \mathbf{2 7 8}, 467$.

Vogt, S. S., 1981, ApJ, 250, 327.

White, S. E., Kundu, M. R. \& Jackson, P. D., 1989, $A \& A$, 225, 112.

White, N. E., Shafer, R. A., Horne, K., Parmar, A. N., \& Culhane, J.L., 1990, ApJ, 350, 776.

Wild, W. J., 1991, $A p J$, 368, 622 .

Zeilik, M., Cox, D., Ledlow, M., Rhodes, M. \& Heckert, P., 1990, ApJ, 363, 647. 


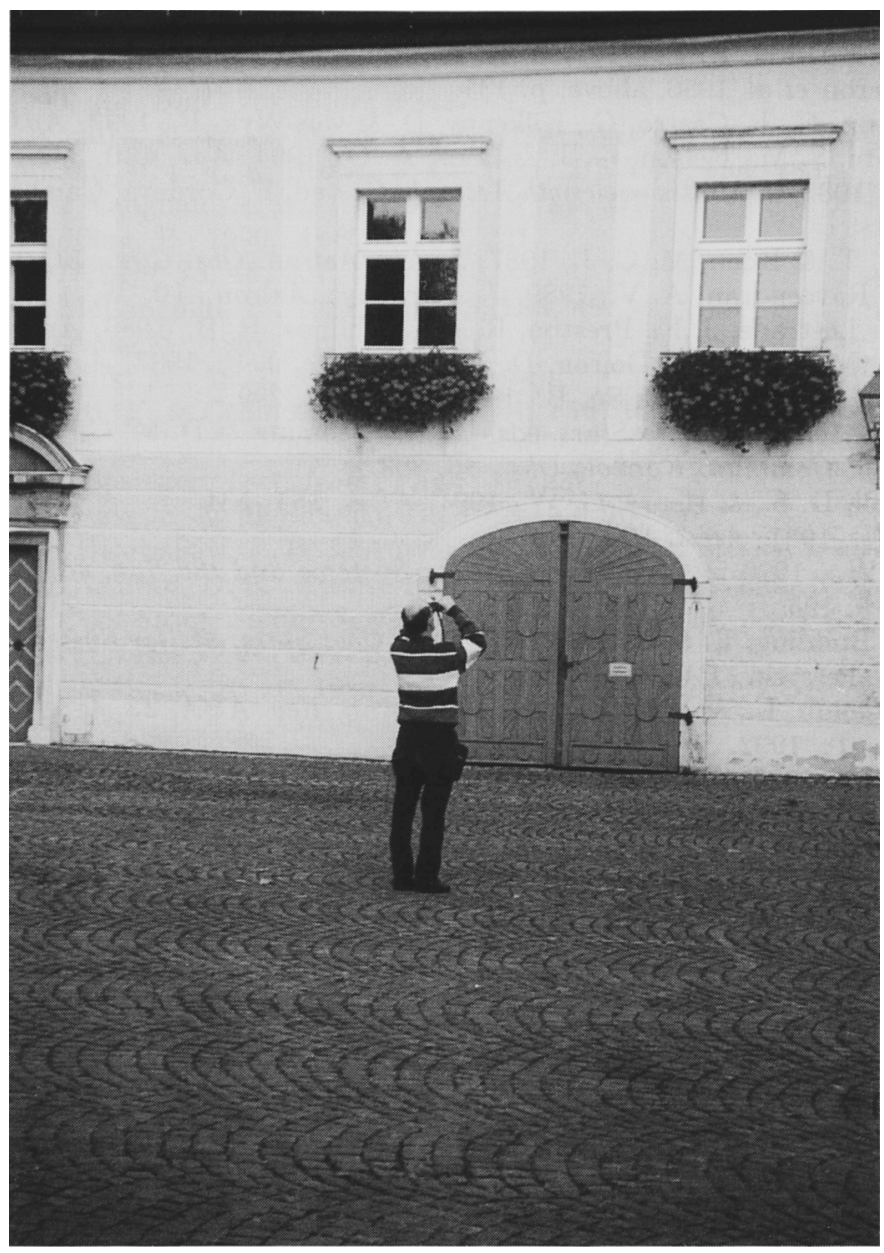

We already emphasized that Frank is a birdwatcher. Proof can be found on a previous page! 